\title{
Stimulation of Cortisol During Mental Task Performance in a Provocative Virtual Environment
}

\author{
Alex H. Bullinger, ${ }^{1,6}$ Ullrich M. Hemmeter, ${ }^{2}$ Oliver Stefani, ${ }^{1}$ \\ Isabelle Angehrn, ${ }^{1}$ Franz Mueller-Spahn, ${ }^{1}$ Evangelos Bekiaris, ${ }^{3}$ \\ Brenda K. Wiederhold, ${ }^{4}$ Hubert Sulzenbacher, ${ }^{5}$ \\ and Ralph Mager ${ }^{1}$
}

\begin{abstract}
Fully immersive and stereoscopic Virtual Environments (VE) represent a powerful multimedia tool for laboratory-based simulations of distinct scenarios including scenarios for evaluating stressful situations resembling reality. Thus far, cortisol secretion as a neuroendocrine parameter of stress has not been evaluated within a Virtual Reality (VR)based paradigm. In this study 94 healthy volunteers were subjected to a provocative VR-paradigm and a cognitive stress task. Provocative in this context means the VE was designed to provoke physiological reactions (cortisol secretion) within the respective users by purpose. It was tested (a) if a fully dynamic VE as opposed to a static VE can be regarded as a stressor and (b) if such a fully dynamic VE can modify an additional response to a cognitive stressor presented within the VE additionally. Furthermore, possible gender-related impacts on cortisol responses were assessed. A significant cortisol increase was observed only after the combined application of the fully dynamic VE and the cognitive stressor, but not after application of the dynamic VE or the cognitive stressor alone. Cortisol reactivity was greater for men than for women. We conclude that a fully dynamic VE does not affect cortisol secretion per se, but increases cortisol responses to a dual task paradigm that includes performance of a stressful mental task. This provides the basis for the application of VR-based technologies in neuroscientific research, including the assessment of the human Hypothalamus-Pituitary-Adrenal (HPA) axis regulation.
\end{abstract}

KEY WORDS: virtual environment; virtual reality; cognitive stress; cortisol; gender.

\footnotetext{
${ }^{1}$ COAT-Basel/UPKBS, Department of Psychiatry, University of Basel, Basel, Switzerland.

${ }^{2}$ Psychiatric University Clinic, University of Marburg, Marburg, Germany.

${ }^{3}$ CERTH/HIT, Centre for Research and Technology Hellas, Athens, Greece.

${ }^{4}$ Virtual Reality Medical Center, San Diego, California.

${ }^{5}$ Psychiatric Clinic, University of Innsbruck, Innsbruck, Austria.

${ }^{6}$ Address all correspondence to Dr. Alex H. Bullinger, MBA, Managing Director, COAT-Basel/UPKBS, Center of Applied Technologies in Neuroscience, Wilhelm Klein-Strasse 27, 4025 Basel, Switzerland; e-mail: bua@ coat-basel.com.
} 


\section{INTRODUCTION}

Fully immersive and stereoscopic Virtual Environments (VE) represent a powerful multimedia tool for laboratory-based simulations of distinct scenarios including scenarios for evaluating stressful situations resembling reality (Bullinger, Rössler, \& Mueller-Spahn, 1998). Therefore, VR-based technologies are of growing interest for the application in different working fields such as industrial design, engineering, rapid prototyping, ergonomics, medicine, and psychology, just to mention the most common ones (Kern \& Breining, 1999). The impact of these technologies on basic research in neuroscience predominantly concerns the simulation of problem solving strategies and the laboratory simulation of stressful situations, either related to an emotional context, such as anxiety disorders or without emotional features (Bullinger, 2001).

An essential component for the development of psychiatric disorders, such as schizophrenia, anxiety, and predominantly depression is an insufficient coping with stressors (Post, 1992). Therefore, the simulation of stressful situations under conditions close to reality may provide an experimental evaluation of stressors and of their effect on the physiological and emotional reactivity in humans under natural conditions.

The most important neurobiological system attacked by stress is the HypothalamusPituitary-Adrenal (HPA) axis (Holsboer, 1999). The release of cortisol, the peripheral indicator of the HPA axis activation during or immediately after stress stimulation, represents an essential mechanism of counterregulation to a dysbalance of the organism from homeostasis (Selye, 1981). Thus, cortisol reactivity reflects a somatic coping mechanism to stress (Frankenhäuser \& Lundberg, 1982; Hemmeter, 2000).

In healthy subjects an activation of the HPA axis induced by somatic or cognitive stressors is described in abundance (e.g., Hemmeter, 2000; Kirschbaum \& Hellhammer, 1989). However, a cortisol response to cognitive stressors has not been consistently found in the studies described in the literature (Berger et al., 1987; Brandenberger, Follenius, Wittersheim, \& Salame, 1980). It is well known though, that the quality of the stressor as well as the duration and intensity of the respective stressor widely affect the cortisol response (Kirschbaum \& Hellhammer, 1989; Vernikos-Danellis \& Heybach, 1980). Major features of stressors that have proven to evoke a cortisol response are loss of control, unpredictability, and novelty (Breier et al., 1987; Dess, Linwick, Patterson, Overmier, \& Levine, 1983; Henry, 1992; Kirschbaum \& Hellhammer, 1989). The cortisol response to a cognitive stress paradigm presented within a VE has not been studied so far.

VR provides a laboratory-based tool to modify not only the context but also the shape of a computer-generated (thus "virtual") three-dimensional space with reality character that may affect the human vestibular system, which is a basic system of human postural control. Cortisol response to a disturbance of the vestibular system has been previously shown in different studies (Dai, Ji, Huang, Sun, \& Dai, 1998; Grigoriev, Nichiporuk, Yasnetsov, \& Shashkov, 1988). Furthermore, the dynamic modification of a VE may appear to be closely associated with novelty and a loss of controllability and therefore should have additional effects on cortisol release. Thus, the observed cortisol responses in these studies may be due to a direct effect on the vestibular system or secondary to the feelings of uncontrollability and novelty within the given context. While the application of a cognitive task alone does not consistently induce an increase of cortisol (Berger et al., 1987; Brandenberger et al., 1980), consistent cortisol increases have been observed after the application of an 
arithmetic task in combination with an uncontrollable and novel demanding situation (for example public speaking) as performed by the Trier Social Stress Test (Kirschbaum, Pirke, \& Hellhammer, 1993) or even with an unconditioned stressor like noise (Brandenberger et al., 1980). Therefore, we suppose that the common application of a cognitive task within a dynamic VE as opposed to a static VE may provide a condition in which an intense cortisol response can be expected.

Thus far, cortisol secretion has not been evaluated in a cognitive stress paradigm combined with VR-based technologies. Therefore, we conducted a study in which we tested a) if the modification of reality induced by dynamic VE as opposed to static VE can be regarded as a stressor and b) if it can modify an additional cognitive stress response. Because a greater cortisol reactivity in response to stress has been reported for men than for women (Gelfin, Lerer, Lesch, Gorfine, \& Allolio, 1995; Kirschbaum, Klauer, Filipp, \& Hellhammer, 1995; Steptoe, Fieldman, Evans, \& Perry, 1996), we were additionally interested in gender differences of the cortisol responses within our study sample. To answer these questions a static and a dynamic VE either with or without a stressful cognitive task was applied to healthy subjects in order to compare cortisol increases in the four resulting groups of subjects.

\section{METHODS}

\section{Study Population}

Initially, the Virtual Environment was designed to examine the impact of VR-induced visual strain on retroretinal blood pressure. Within this experiment 160 healthy subjects were tested under naturalistic conditions, recruited at a public desk of the Ophtalmological University Clinic of Basel, Switzerland, during a trade fair in Basel. In a subsample of these volunteers saliva samples for the assessment of cortisol could be obtained. After subjects agreed to participate and signed informed consent, they were immediately examined in a quiet adjacent room. Complete data sets on cortisol secretion within this experiment were obtained from 94 healthy subjects ( 37 women $46.4 \pm 12.6$ and 57 men, $46.4 \pm 15.3$ years, range 16-82) whose data are presented here.

Participants were screened by a previous interview for somatic or mental health-related diseases and heredity of mental disorders. Shift workers and subjects on medications or with a transmeridian flight within the last 3 weeks were excluded, as well as participants with an interpupillary distance (IPD) not matching adjustment levels of the binocular data helmet (Head mounted display, HMD). All participants were screened for their familiarity with computer systems. In case of experience with HMDs or any other kind of stereoscopic, fully immersive projection technologies, participants were also excluded from the study. In addition, only people with a regular pattern of sleep and food intake were allowed to participate. People wearing glasses were not excluded, because glasses could be worn under the HMD without any problems.

\section{Experimental Conditions}

In all conditions a binocular data helmet (head mounted display, HMD) for the application of the VR task was adapted to the participants showing a three-dimensional room, 
which was not modified in order to control for a potential stress reaction induced by mere wearing of the helmet.

All participants were randomly divided in four different groups:

Group 1. A control condition using a static Virtual Environment (VE), without presentation of a cognitive stressor.

Group 2. A concentration speed task including aspects of divided attention (cognitive stress paradigm), also using the static VE.

Group 3. Using a fully dynamic VE, that performed yaw, pitch, and roll movements with a frequency of $2 \mathrm{~Hz}$, without presentation of a cognitive stressor.

Group 4. Using the same dynamic VE as in group 3, combined with the cognitive stress paradigm used in group 2.

\section{Static vs. Dynamic Virtual Environment}

The virtual environment showed a rectangular three-dimensional room, including some furniture and featuring a window with a view into a garden (see Fig. 1). While used as static VE (groups 1 and 2), the room did not move at all, whereas used as a dynamic VE (group 3 and 4), the room performed yaw, pitch, and roll movements with a frequency of $2 \mathrm{~Hz}$ and an amplitude of $1.2 \mathrm{~m}$.

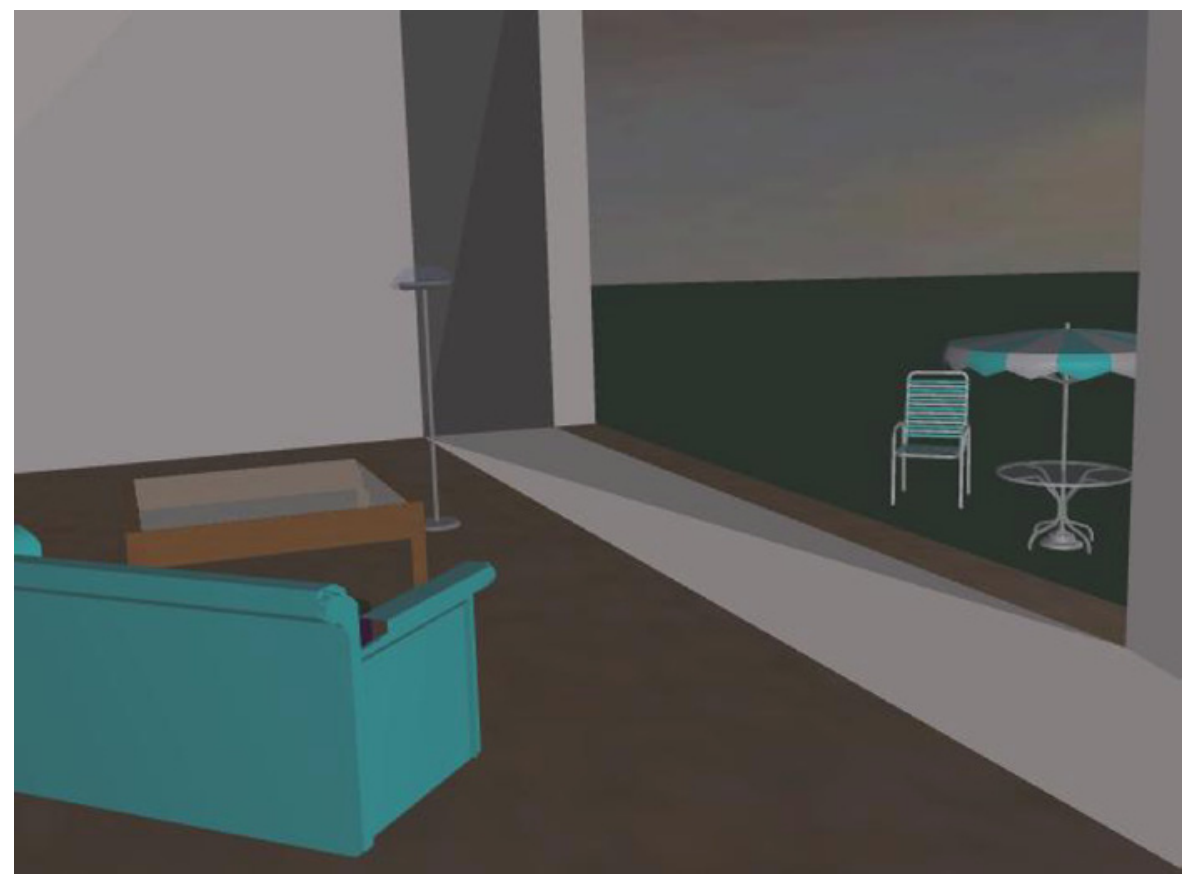

Fig. 1. Virtual room used as experimental paradigm. 


\section{Description of the VR-System}

The VR-system used in this study was a Silicon Graphics ${ }^{\circledR}$ Onyx $^{2}$, Infinite Reality graphics subsystem with 8-channel multichannel option, 2 CPUs (MIPS R10000), $256 \mathrm{MB}$ RAM, and 1 raster manager with $64 \mathrm{MB}$ texture memory and $80 \mathrm{MB}$ frame buffer. As real-time tracking device an Ascension ${ }^{\circledR}$ Flock of Birds was used, while a MIKE-built by Fraunhofer IAO, Stuttgart—served as input device.

\section{Cognitive Stress Condition}

A speed concentration task including aspects of divided attention and visual scanning designed for the application in VR had to be performed by subjects in the cognitive stress cognition task. There were three three-dimensional items randomly appearing within the participants' field of vision while wearing the data helmet (HMD, Fig. 2): Black appearing objects required no actions from the participants, regardless of the shape of the object. In contrast, colored objects (pyramids and squares in yellow, red, or green) required the participants to press the upper button of the input device, while gray figures required the participants to press the lower button of the input device. Subjects were advised to react as fast and exactly as possible. Pretests indicated this paradigm as quite stressful.

All participants received extensive written explanations about the general aims of the study, the four different experimental groups, the procedure of random assignment to the experimental groups, the elements and requirements of the cognitive stress paradigm as well as an oral introduction explaining to them the components of the VR-System. Participants were informed about all possible experimental conditions prior to the start of the experiment, but they did not know in which of the four conditions they participated. Informed consent was obtained from all participants.

\section{Dependent Variables}

The target parameter of this study was the assessment of cortisol secretion in saliva measured before and after the stimulation by VR (with or without cognitive stress). Cortisol

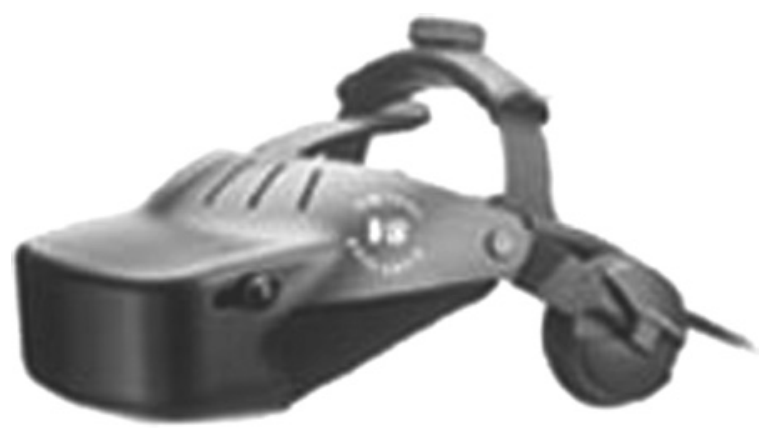

Fig. 2. Head-mounted display (HMD) used during this study (manufacturer: Virtual Research). 
was assessed: twice at baseline, after the arrival of the subjects and immediately before the start of the experimental conditions (dynamic VR \pm , cognitive stress \pm ), and twice after the stimulation (response measurements), the first measurement directly after the end of the procedure, the second one $15 \mathrm{~min}$ later. Saliva was collected by salivettes, frozen by $-20^{\circ} \mathrm{C}$, and analyzed by a standard RIA-method as described elsewhere (Hennig, Lange et al., 2000; Hennig, Toll et al., 2000). All tests were conducted in the afternoon between 2 and 5 p.m. and lasted $20 \mathrm{~min}$.

\section{Statistics}

Based on the combined within/between-subjects design, which consisted of two between-subjects factors (dynamic VE $\mp$, cognitive stress $\mp$ ) and one within-subjects factor (time) an analysis of covariance (ANCOVA) for repeated measurements was performed with $\mathrm{T} 1$ as the covariate and T2 (baseline), and the response values T3 (=R1) and T4 (=R2) as three levels of the factor time. In case of significant main or interaction effects, subsequent $t$-tests for the location of effects were performed. When comparing two response values with the baseline value, the $\alpha$-level was Bonferroni corrected and set to $p \leq .025$.

For the evaluation of differences between males and females an additional analysis of covariance was performed with gender as an additional independent factor. Additionally, two ANCOVAs corresponding to that in the total sample were performed separately for men and women.

\section{RESULTS}

As a result of the wide variation of cortisol baseline values and a significant difference in baseline values between participants in the control condition and participants who had to deal with the stress conditions, an analysis of covariance was performed using T1 as the covariate, as outlined in the statistics section.

This procedure revealed a significant three-way interaction $(d f=2,180 ; F=3.96$; $p=.02)$. After the combined application of both stressors, the cognitive stress paradigm and the dynamic VR-paradigm at T4 ( $t$-test; $t=-2.79, p \leq .01)$, there was a significant increase of cortisol and a trend of an increase at T3 ( $t$-test, $t=-2,18, p \leq .05$, Table I, Fig. 3), in contrast to the stimulation with the application of either the dynamic VR or the cognitive stress paradigm alone and to the control condition (Table I, Fig. 4).

\section{Effects of Gender and Age}

The inclusion of gender as an additional between-subjects factor into the ANCOVA described above did not reveal any significant main or interaction effects of gender, while the previously obtained three-way interaction effect was still present in this 4-factorial ANCOVA $(d f=172,2, F=4.5, p \leq .01)$.

However, when performing the analysis in males and females separately, the threeway interaction effect was only significant in females $(d f=66,2, F=4.4, p \leq .01)$. In 
Table I. Cortisol Secretion (nmol/l) in Virtual Reality (VR, Static vs. Dynamic) and Cognitive Stress $(\mathrm{CT}, \mp)$, Total Sample $(N=94)$

\begin{tabular}{|c|c|c|c|c|}
\hline & $\begin{array}{c}\text { Cortisol } \\
\text { baseline } 2\end{array}$ & $\begin{array}{c}\text { Cortisol } \\
\text { reaction } 1\end{array}$ & $\begin{array}{l}\text { Cortisol } \\
\text { reaction } 2\end{array}$ & $\begin{array}{c}\text { Covariate cortisol } \\
\text { (baseline } 1 \text { ) }\end{array}$ \\
\hline Controls $(N=16)$ & $2.69 \pm 1.34$ & $3.07 \pm 2.03$ & $3.30 \pm 2.06$ & $2.35 \pm 1.49$ \\
\hline Cognitive task $(N=27)$ & $3.19 \pm 1.75$ & $2.88 \pm 1.73$ & $3.03 \pm 1.61$ & $2.76 \pm 2.02$ \\
\hline Dynamic VR $(N=27)$ & $3.07 \pm 1.84$ & $2.58 \pm 1.71$ & $2.80 \pm 2.22$ & $1.91 \pm 1.20$ \\
\hline Both stressors $(N=24)$ & $2.39 \pm 0.90$ & $3.25 \pm 1.73^{*}$ & $3.66 \pm 2.04^{* *}$ & $2.90 \pm 1.51$ \\
\hline \multicolumn{5}{|l|}{ ANCOVA } \\
\hline Source of variation & & $d f$ & $F$ & $p$ \\
\hline Cognitive task $(-;+)$ & & $1 ; 88$ & 0.197 & $n s$ \\
\hline VR (static; dynamic) & & $1 ; 88$ & 0.003 & $n s$ \\
\hline Time & & $2 ; 180$ & 1.337 & $n s$ \\
\hline Cognitive task $\times$ VR & & $1 ; 88$ & 0.016 & $n s$ \\
\hline Cognitive task $\times$ time & & $2 ; 180$ & 0.42 & $n s$ \\
\hline $\mathrm{VR} \times$ time & & $2 ; 180$ & 0.18 & $n s$ \\
\hline $\mathrm{CT} \times \mathrm{VR} \times$ time & & $2 ; 180$ & 3.958 & .02 \\
\hline
\end{tabular}

both females and males cortisol increased in the combined dynamic VE/cognitive stress condition (Table II).

On a descriptive level it may be added that in men cortisol increased also in the dynamic $\mathrm{VE}$ and remained constant in the cognitive stress paradigm, whereas in women cortisol dropped in both these conditions. In the control condition cortisol was nonsignificantly increased in both, men and women (Table II).

No significant correlations between cortisol and age were discovered in this study.

\section{DISCUSSION}

This study evaluates for the first time the effects of an activation paradigm based on Virtual Reality - technology on the neuroendocrine stress reaction of cortisol. The main result of this study is that a dynamic variation of the Virtual Environment focusing on the modification of the controllability of the vestibular system in combination with a cognitive stress paradigm that included a divided attention speed task within the VE was able to induce a significant activation of the HPA axis. In contrast, the mere application of the

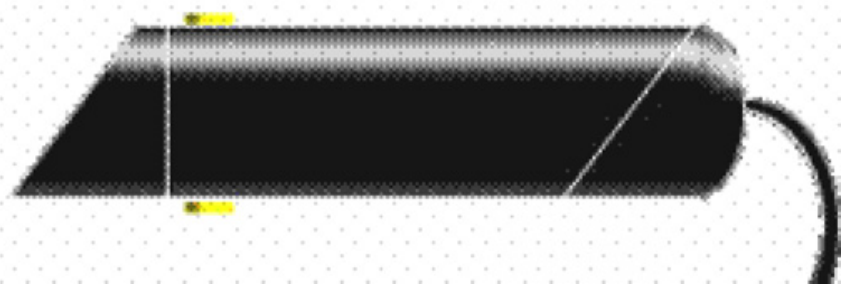

Fig. 3. (Schematic) Input device used within this study ("MIKE," manufacturer: FhG-IAO, Stuttgart, Germany). 


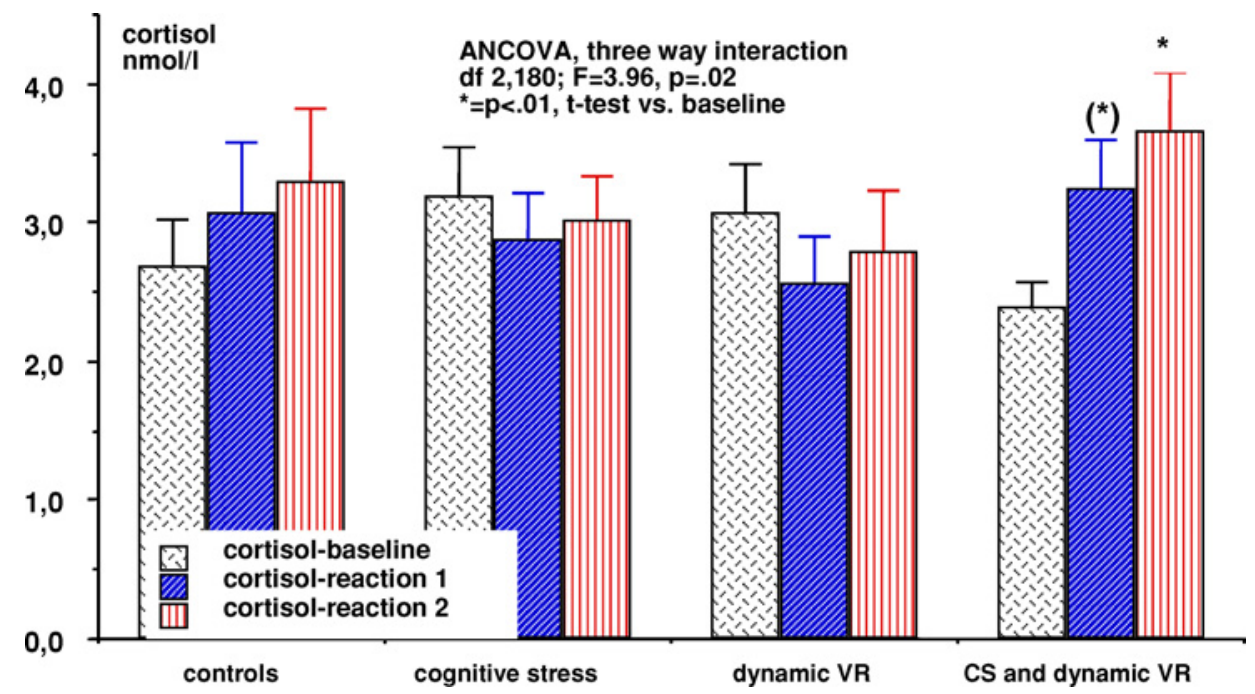

Fig. 4. Cortisol responses after cognitive stress (CS) and/or dynamic virtual reality in healthy subjects (total group).

cognitive paradigm in a static VE and the modification of the virtual environment without cognitive stress did not lead to cortisol release in the total group. The latter finding may indicate that the intensities of the two stressors, the cognitive task and the dynamic VE condition, might have been too weak for a substantial cortisol release.

An explanation for the cortisol increase observed in the combination of cognitive stress and dynamic VE may be provided by the different levels of controllability between the single stress conditions and the combination of both. The application of each stressor alone may not be able to induce a loss of control in the subjects. Therefore, subjects can easily cope with these conditions (Breier et al., 1987; Frankenhäuser, Lundberg, \& Forsman, 1980; Lundberg, 1984). In terms of the application of VE alone, a mere modulation of the virtual environment that has an impact on the vestibular system is not sufficient to induce a neuroendocrinological stress response of cortisol. However, the additional application of dynamic VE during a cognitive stressor may reduce the controllability of the cognitive stress condition, and therefore, evoke the observed cortisol response. The combined application of the cognitive stressor and the dynamic VE may resemble the consistent cortisol stimulation observed in the TSST, which also includes a mental and an emotional stressor including aspects of uncontrollability and novelty (Kirschbaum et al., 1993).

Unfortunately, we did not assess subjects' feelings of uncontrollability and further psychological items within this study, because subjects being visitors at a trade fair were only available for a short period of time. This shortcoming was due to the naturalistic conditions of this study.

But there are two further factors, which have to be taken into account. The first one is the fact that subjects were faced with the VR-control condition (application of the helmet and presentation of a virtual room) and the second one is the fact of the circadian secretion of cortisol. As no control assessment of cortisol without the application of the helmet and the presentation of the virtual room had been carried out, we are not aware of the true 
Table II. Cortisol Secretion (nmol/l) in Virtual Reality (VR, Static vs. Dynamic) and Cognitive Stress $(\mathrm{CT}, \mp)$

\begin{tabular}{|c|c|c|c|c|}
\hline & $\begin{array}{c}\text { Cortisol } \\
\text { baseline } 2\end{array}$ & $\begin{array}{l}\text { Cortisol } \\
\text { reaction } 1\end{array}$ & $\begin{array}{l}\text { Cortisol } \\
\text { reaction } 2\end{array}$ & $\begin{array}{l}\text { Covariate cortisol } \\
\quad \text { (baseline } 1)\end{array}$ \\
\hline \multicolumn{5}{|l|}{ Females $(N=37)$} \\
\hline Controls $(N=7)$ & $2.41 \pm 1.15$ & $2.61 \pm 1.18$ & $2.96 \pm 1.40$ & $1.67 \pm 0.43$ \\
\hline Cognitive task $(N=10)$ & $3.74 \pm 2.12$ & $2.68 \pm 1.34$ & $3.29 \pm 1.78$ & $3.15 \pm 2.89$ \\
\hline Dynamic VR $(N=12)$ & $3.22 \pm 1.50$ & $2.02 \pm 1.02$ & $1.86 \pm 0.93$ & $1.78 \pm 0.84$ \\
\hline Both stressors $(N=8)$ & $2.71 \pm 1.20$ & $3.41 \pm 2.05^{*}$ & $4.20 \pm 2.75^{*}$ & $3.03 \pm 1.50$ \\
\hline \multicolumn{5}{|l|}{ ANCOVA } \\
\hline Source of variation & & $d f$ & $F$ & $p$ \\
\hline Cogn task $(-;+)$ & & $1 ; 31$ & 2.076 & ns \\
\hline VR (static; dynamic) & & $1 ; 31$ & 0.007 & $n s$ \\
\hline Time & & $2 ; 66$ & 0.775 & $n s$ \\
\hline Cognitive task $\times$ VR & & $1 ; 31$ & 0.707 & $n s$ \\
\hline Cognitive task $\times$ time & & $2 ; 66$ & 0.921 & $n s$ \\
\hline $\mathrm{VR} \times$ time & & $2 ; 66$ & 0.046 & $n s$ \\
\hline $\mathrm{CT} \times \mathrm{VR} \times$ time & & $2 ; 66$ & 4.349 & .02 \\
\hline \multicolumn{5}{|l|}{ Males $(N=57)$} \\
\hline Controls $(N=15)$ & $2.90 \pm 1.52$ & $3.43 \pm 2.52$ & $3.56 \pm 2.51$ & $2.89 \pm 1.82$ \\
\hline Cognitive task $(N=12)$ & $2.88 \pm 1.47$ & $2.30 \pm 1.95$ & $2.88 \pm 1.54$ & $2.54 \pm 1.33$ \\
\hline Dynamic VR $(N=16)$ & $2.95 \pm 2.11$ & $3.02 \pm 2.03$ & $3.55 \pm 2.66$ & $2.03 \pm 1.45$ \\
\hline Both stressors $(N=14)$ & $2.24 \pm 0.71$ & $3.18 \pm 1.61$ & $3.39 \pm 1.62$ & $2.83 \pm 1.56$ \\
\hline \multicolumn{5}{|l|}{ ANCOVA } \\
\hline Source of variation & & $d f$ & $F$ & $p$ \\
\hline Cognitive task $(-;+)$ & & $1 ; 51$ & 1.642 & ns \\
\hline VR (static; dynamic) & & $1 ; 51$ & 0.068 & $n s$ \\
\hline Time & & $2 ; 106$ & 2.164 & $n s$ \\
\hline Cognitive task $\times$ VR & & $1 ; 51$ & 0.426 & $n s$ \\
\hline Cognitive task $\times$ time & & $2 ; 106$ & 0.124 & $n s$ \\
\hline $\mathrm{VR} \times$ time & & $2 ; 106$ & 0.434 & $n s$ \\
\hline $\mathrm{CT} \times \mathrm{VR} \times$ time & & $2 ; 106$ & 0.735 & $n s$ \\
\hline
\end{tabular}

${ }^{*} p<.05, t$-test vs. baseline, data represent raw values.

circadian pattern of cortisol in these subjects. It is well known that cortisol values drop in the afternoon hours under resting conditions to a basal level (Sachar, Hellman, \& Fukushima, 1970). Thus, it can be concluded that the invariant cortisol secretion under each of the isolated stress conditions may nevertheless reflect a moderate stress effect in comparison to the (unknown) circadian decline. This suggestion contrasts with the observation of the slight nonsignificant cortisol increase in the control condition (without dynamic VE or cognitive stress). A probable explanation for this finding may be provided by differences in the level of anticipation, because subjects were in a novel situation, unaware of things to come. In contrast to this condition, in both of the isolated activation conditions subjects were faced with requirements they were able to cope with, while in the control condition subjects could have rested in expectancy, waiting that something new will occur. This situation includes aspects of novelty and anticipation, which have been shown to belong to the most potent and distinct features of stressors stimulating cortisol secretion (Kirschbaum \& Hellhammer, 1989; Mason, 1975a, 1975b).

Based on the assumption that there was already in the control condition a certain amount of subjectively experienced stress, which is reflected by the lack of the expected circadian cortisol decrease, it can be concluded that-within this experiment in generalthe HPA axis presented with an increased tonic activation which augmented the inhibitory 
effect of feedback regulation. This may prevent a cortisol rise during the additional isolated stress either by the cognitive stressor or by modification of the virtual environment. A similar phenomenon of an inhibition of cortisol during an additional mental stressor applied during an already activated cortisol secretion because of fasting has been observed previously in healthy subjects (Hemmeter, Burkhard, Shaye, Holsboer-Trachsler, \& Netter, 1999; Kirschbaum et al., 1997).

The intensification of the stress situation by the combined application of both stressors (dynamic VE and cognitive task) may reach a level that overrides the inhibitory effects which are active under low dose stress in the control condition, and, therefore, result in a significant cortisol response. A theoretical basis for this suggestion is provided by the stress concept of the general adaptation syndrome (Selye, 1951), which predicts increasing cortisol in the later phase of stress stimulation, which is either chronic or substantially intense.

The cortisol secretion in this experiment appears-at least in part-to be modified by gender. Although no significant main or interaction effects of gender were detected in the 4-way ANCOVA, males as well as females responded by an increase of cortisol in the combined stress application. But, the reaction to the isolated stress paradigms was different in men and women. The cortisol secretion in women declined upon the isolated presentation of the stressors, whereas in men cortisol was slightly increased in the dynamic $\mathrm{VE}$ - condition and remained constant in the cognitive stress paradigm. The lack of a significant three-way interaction in men as opposed to women and of a significant four-way interaction with gender in the total group may be explained by the additional tendency of men to react by a cortisol increase in the dynamic VR applied alone and in part also in the isolated cognitive stress paradigm. These findings are consistent with results of previous studies on gender differences in cortisol secretion to stimulation with one stressor, which show a greater reactivity for men than for women (Gelfin et al., 1995; Kirschbaum et al., 1995; Steptoe et al., 1996). This may explain the different, but nonsignificant cortisol responses in men compared to women in the isolated stimulation paradigms. An explanation of the sex differences in cortisol responses may be provided by a higher inhibitory effect of basal cortisol on the HPA axis in females (Heuser et al., 1994). A final conclusion about the underlying mechanisms of the different cortisol reactions to the experimental conditions in all subjects and about the differences between men and women is not possible with the data at hand, mainly because different factors additionally affecting cortisol secretion have not been sufficiently controlled due to the open character of the study.

In particular, somatic and personality traits, which have been proven to explain a certain interindividual variability of the cortisol reaction (Hemmeter, 2000; Netter, Hennig, \& Huwe, 1997; Netter, Hennig, \& Rohrmann, 1999), have not been assessed. Last but not least, subjective stress perception and ego-involvement in the study were not systematically evaluated in this study, which was performed in a natural environment. In spite of these shortcomings, the modification of the visual impression of reality by the Virtual Environment has been shown to alter cortisol secretion during a task with which subjects can cope physiologically in undisturbed conditions.

We conclude that the application and modification of a Virtual Environment as such does not result in an increase of cortisol. However, tasks requiring the modification of subjective reality impression combined with a demanding mental task seem to induce the neuroendocrine secretion of cortisol. The cortisol response within the virtual paradigm is 
highly dependent on the kind of stimulation and context. Thus, it provides the basis for the application of VR-based technologies in neuroscientific research, which includes the assessment of the human HPA axis regulation. The impact of this technology for monitoring diagnostic and therapeutic procedures in psychiatric disorders related to stress has to be determined in further studies controlling more of the additional intervening variables that were not controlled during the study presented here.

\section{REFERENCES}

Berger, M., Bossert, S., Krieg, J. C., Dirlich, G., Ettmeier, W., Schreiber, W., \& von Zerssen, D. (1987). Interindividual differences in the susceptibility of the cortisol system: An important factor for the degree of hypercortisolism in stress situations? Biological Psychiatry, 22, 1327-1339.

Brandenberger, G., Follenius, M., Wittersheim, G., \& Salame, P. (1980). Plasma catecholamines and pituitary adrenal hormones related to mental task demand under quiet and noise conditions. Biological Psychology, 10, 239-252.

Breier, A., Albus, M., Pickar, D., Zahn, T. P., Wolkowitz, O. M., \& Paul, S. (1987). Controllable and uncontrollable stress in humans: Alterations in mood and neuroendocrine and psychophysiological function. American Journal of Psychiatry, 144, 1419-1425.

Bullinger, A. H. (2001). VE user physiological, cognitive and psychological needs. Deliverable to the European Commission, Contract No. IST-2000-26089, pp. 1-98.

Bullinger, A. H., Rössler, A., \& Mueller-Spahn, F. (1998). From toy to tool: The development of immersive virtual reality environments for psychotherapy of specific phobias. In G. Riva (Ed.), Virtual reality in clinical psychology (pp. 103-111). Amsterdam: IOS.

Dai, Y., Ji, G., Huang, Y., Sun, X., \& Dai, F. (1998, April) Changes of plasma endocrine hormone in pilots under Coriolis acceleration. Space Medicine and Medcal Engineering (Beijing), 11(2), 121-123.

Dess, N. K., Linwick, D., Patterson, J., Overmier, J. B., \& Levine, S. (1983). Immediate and proactive effects of controllability and predictability on plasma cortisol responses to shocks in dogs. Behavioral Neuroscience, 97(6), 1005-1016.

Frankenhäuser, M., \& Lundberg, U. (1982). Psychoneuroendocrine aspects of effort and distress as modified by personal control. In W. Bachmann \& I. Udris (Eds.), Mental load and stress in activity European approaches (pp. 97-103). Amsterdam: North-Holland.

Frankenhäuser, M., Lundberg, U., \& Forsman, L. (1980). Dissociation between sympathetic-adrenal and pituitaryadrenal responses to an achievement situation characterized by high controllability: Comparison between type A and type B males and females. Biological Psychology 10(2), 79-91.

Gelfin, Y., Lerer, B., Lesch, K. P., Gorfine, M., \& Allolio, B. (1995). Complex effects of age and gender of hypothermic adrenocorticotrophic hormone and cortisol responses to ipsapirone challenge in normal subjects. Psychopharmacology, 120(3), 356-364.

Grigoriev, A. I., Nichiporuk, I. A., Yasnetsov, V. V., \& Shashkov, V. S. (1988, April). Hormonal status and fluid electrolyte metabolism in motion sickness. Aviation Space and Environmental Medicine, 59(4), 301-305.

Hemmeter, U. (2000). Der Einfluss der Persönlichkeitsdisposition auf die Cortisolreaktion nach experimentellem Stress und Fasten - ein psycho-neuroendokrinologischer Ansatz der differentiellen Reagibilität der Persönlichkeit auf Stress, Psychologische Forschungsergebnisse, 343 S., Kovacs Verlag, Hamburg, Germany.

Hemmeter, U., Burkhard, H., Shaye, R., Holsboer-Trachsler, E., \& Netter, P. (1999). Blunted cortisol response to different stressors in subjects scoring high on depression related personality traits. Pharmacopsychiatry, 32, 185.

Hennig, J., Lange, N., Haag, A., Rohrmann, S., \& Netter, P. (2000, September) Reboxetine in a neuroendocrine challenge paradigm: Evidence for high cortisol responses in healthy volunteers scoring high on subclinical depression. International Journal of Neuropsychopharmacology, 3(3), 193-201.

Hennig, J., Toll, C., Schonlau, P., Rohrmann, S., \& Netter, P. (2000) Endocrine responses after d-fenfluramine and ipsapirone challenge: Further support for Cloninger's tridimensional model of personality. Neuropsychobiology, 41(1), 38-47.

Henry, J. P. (1992). Biological basis of stress response. Integrated Physiological and Behavioral Science, 27(1), 66-83.

Heuser, I. J., Gotthardt, U., Schweiger, U., Schmider, J., Lammers, C. H., Dettling, M., \& Holsboer, F. (1994, March-April) Age-associated changes of pituitary-adrenocortical hormone regulation in humans: Importance of gender. Neurobiology of Aging, 15(2), 227-231. 
Holsboer, F. (1999). The rationale for corticotropin-releasing hormone receptor (CRH-R) antagonists to treat depression and anxiety. Journal of Psychiatric Research, 33, 181-214.

Kern, P., \& Breining, R. (1999). Experiences with interfaces for Virtual Environments in the field of engineering. In H.-J. Bullinger \& J. Ziegler (Eds.), Human-Computer Interaction: Communication, Cooperation, and Application Design (Vol. 2), Proceedings of the 8th International Conference on Human-Computer Interaction (pp. 696-700). Mahwah, NJ: Erlbaum.

Kirschbaum, C., Gonzalez-Bono, E., Rohleder, N., Gessner, C., Pirke, K. M., Salvador, A., \& Hellhammer, D. H. (1997). Effects of fasting and glucose load on free cortisol responses to stress and nicotine. Journal of Clinical Endocrinology and Metabolism, 82(4), 1101-1105.

Kirschbaum, C., \& Hellhammer, D. H. (1989). Salivary cortisol in psychobiological research: An overview. Neuropsychobiology, 22, 150-169.

Kirschbaum, C., Klauer, T., Filipp, S. H., \& Hellhammer, D. H. (1995). Sex-specific effects of social support on cortisol and subjective responses ot acute psychological stress. Psychosomatic Medicine, 57(1), 23-31.

Kirschbaum, C., Pirke, K. M., \& Hellhammer, D. H. (1993). The "Trier Social Stress Test”- a tool for investigating psychobiological stress responses in a laboratory setting. Neuropsychobiology, 28(1-2), 76-81.

Lundberg, U. (1984). Human psychobiology in Scandinavia: II. Psychoneuroendocrinology-human stress and coping processes. Scandinavian Journal of Psychology, 25, 214-226.

Mason, J. W. (1975a). Emotion as reflected in patterns of endocrine integration. In L. Levi (Ed.), Emotions: Their parameters and measurement. New York: Raven.

Mason, J. W. (1975b). A historical view of the "stress" field, part I and II. Journal of Human Stress, 1(6-12), 22-36.

Netter, P., Hennig, J., \& Huwe, S. (1997, October) Explanatory models of anxiety from the viewpoint of biopsychology and pharmacopsychology. Anesthesiologie Intensivmedizin Notfallmedizin Schmerztherapie, 32 (Suppl. 3), S325-S329.

Netter, P., Hennig, J., \& Rohrmann, S. (1999, January). Psychobiological differences between the aggression and psychoticism dimension. Pharmacopsychiatry, 32(1), 5-12.

Post, R. M. (1992). Transduction of psychosocial stress into the neurobiology of recurrent affective disorder. American Journal of Psychiatry, 149, 999-1010.

Sachar, E. J., Hellman, L., \& Fukushima, D. K. (1970). Cortisol production in depressive illness. Archives of General Psychiatry, 43, 878-884.

Selye, H. (1951). Das allgemeine Adaptationssyndrom als Grundlage für eine einheitliche Theorie der Medizin. Medizinische Wochenschrift, 70, 965-967, 1001-1003.

Selye, H. (1981). Geschichte und Grundzüge des Streßkonzepts. In J. R. Nitsch (Ed.), Streß. (pp. 161-187). Bern, Switzerland: Hans Huber.

Steptoe, A., Fieldman, G., Evans, O., \& Perry, L. (1996). Cardiovascular risk and responsivity to mental stress: The influence of age, gender, and risk factors. Journal of Cardiovascular Risk, 3(1), 83-93.

Vernikos-Danellis, J., \& Heybach, J. P. (1980). Psychophysiologic mechanisms regulating the hypothalamicpituitary-adrenal response to stress. In H. Selye (Ed.), Selye's guide to stress research (Vol. 1, pp. 206-251). Torornto. 